\title{
The creation of a national coalition to target adolescent idiopathic scoliosis: a meeting report
}

This article was published in the following Dove Medical Press journal:

Adolescent Health, Medicine and Therapeutics

\author{
Milena Cioana ${ }^{1,2}$ \\ Devin Peterson ${ }^{3}$ \\ Paul Missiuna ${ }^{3}$ \\ Ron El-Hawary ${ }^{4}$ \\ Timothy Carey ${ }^{5,6}$ \\ Murray A Potter 7 \\ Laura Banfield ${ }^{8}$ \\ Lehana Thabane ${ }^{9-12}$ \\ M Constantine Samaan ${ }^{1,2,9}$
}

'Department of Pediatrics, McMaster University, Hamilton, ON, Canada; ${ }^{2}$ Division of Pediatric Endocrinology, McMaster Children's Hospital, Hamilton, ON, Canada; ${ }^{3}$ Division of Orthopedics, Department of Surgery, McMaster University, Hamilton, ON, Canada; ${ }^{4}$ Orthopedic Surgery Department, IWK Children's Health Center, Dalhousie University, Halifax, NS, Canada;

${ }^{5}$ Department of Pediatrics, Schulich School of Medicine and Dentistry, University of Western Ontario, London, ON, Canada ${ }^{6}$ Department of Surgery, Schulich School of Medicine and Dentistry, Western University, London, ON, Canada; ${ }^{7}$ Department of Pathology and Molecular Medicine, McMaster University, Hamilton, ON, Canada; ${ }^{8}$ Health Sciences Library, McMaster University, Hamilton, ON, Canada; 'Department of Health Research Methods, Evidence and Impact, McMaster University, Hamilton, ON, Canada; ${ }^{10}$ Department of Anesthesia, McMaster University, Hamilton, ON, Canada; "Centre for Evaluation of Medicines, St Joseph's Health Care, Hamilton, ON, Canada; '2Biostatistics Unit, St Joseph's Healthcare-Hamilton, Hamilton, ON, Canada

Correspondence: M Constantine Samaan Department of Pediatrics, McMaster University, 1280 Main Street West, HSC-3A, Hamilton, ON L8S 4KI, Canada Tel +l 9055212100 ext 75926 Email samaanc@mcmaster.ca

\begin{abstract}
In this report, we document the discussions and recommendations of a national conference designed to create a coalition to tackle adolescent idiopathic scoliosis (AIS) held on June 6 and 7, 2017 in Hamilton, ON, Canada. The goal of the establishment of this coalition is to join the efforts of patients, parents, physicians, researchers and other stakeholders to identify stakeholders' perspectives and to categorize gaps in knowledge and target further AIS research and clinical care priorities. The participants' main priorities included focus on shared decision making regarding clinical and research priorities between the stakeholders on the clinical, research and policy sides with patients and families. In addition, improvements in the dissemination of information via digital platforms and identification of cost-effective screening strategies that may help early identification and intervention were also recognized as a priority. Commitment was reached to form a national coalition to understand the determinants of this condition and enhance patient outcomes through improved clinical care and research efforts.
\end{abstract}

Keywords: adolescent idiopathic scoliosis, childhood scoliosis, coalition, pediatric

\section{Introduction}

Adolescent idiopathic scoliosis (AIS) is the commonest pediatric spinal deformity affecting millions of children worldwide, with a prevalence of up to $3 \%$. $^{1}$ The deformity can affect the quality of life of patients across their lifespan and has been associated in some cases with pain, mobility limitations, pulmonary hypertension and psychopathology. ${ }^{2-4}$ Treatment options have been stratified based on the severity of the spinal curving and include observation for mild curves and bracing or surgery for more advanced curves. ${ }^{1}$ While these treatments can limit the progression or partially correct scoliosis, they can be associated with neurological complications, worsening pain and decreased mobility. ${ }^{5-7}$

Many potential etiologies have been proposed to explain AIS including genetic, epigenetic, mechanical, neurophysiological, metabolic and environmental factors. ${ }^{8,9}$ However, none of these pathways offer a conclusive explanation for the development and progression of spinal changes noted in AIS, and it is likely that some or all of them contribute to the pathogenesis of AIS. It is crucial to ascertain the mechanisms of AIS, as this will inform the design of interventions, improve health outcomes and reduce morbidities and health care system utilization.

Understanding the diverse mechanisms that are likely driving AIS require the incorporation of transdisciplinary partnerships. In addition, the creation of cohorts of sufficient sample size, and the provision of resources and infrastructure to apply state-of-the-art 
science, rigorous methodologies and analyses to make sound conclusions are all needed to improve health outcomes. In addition, these findings must be effectively communicated to decision makers in charge of health care resource allocation. Hence, stakeholder involvement should be a pillar in the development of these initiatives and should include patients, families, physicians, allied health clinicians, researchers and policymakers to strengthen clinical care and the conduct of comprehensive studies and to help address the most urgent questions in the field of AIS research.

To help develop an understanding of the perspectives of different stakeholders and to define a research agenda, a national conference was organized to help build a national coalition to target the future of AIS research and clinical care.

\section{Methods}

This national conference was held on June 6 and 7, 2017 in Hamilton, ON, Canada. The conference brought together AIS stakeholders including patients, parents, students, clinicians and researchers.

The fundamental goals of the meeting were to:

1. Understand patient and family perspectives in studying AIS

2. Identify the research priority areas in AIS

3. Define the structure of a national coalition to tackle AIS

The conference combined presentations and roundtable discussions to define an agenda for the coalition. Day 1 involved presentations related to the current landscape of AIS research, including epidemiological and mechanistic studies that have studied patients with AIS. Day 2 was focused on discussions regarding the development of the coalition.

\section{Results}

The conference was attended by 23 participants $(n=13$ male) from four pediatric academic centers across Canada, including patients with family members $(n=3)$, physicians/ researchers $(n=9)$, students $(n=10)$ and one health sciences librarian with research expertise in the design of database search strategies.

\section{Understanding patient and family perspectives in studying AIS}

There were several priorities that were identified from the discussions during this meeting. Patient engagement in shared decision making regarding care was highlighted. It was agreed that there is a need to develop a national platform to engage patients and families in the development of the format of how this can be done.

There were discussions about improvements in the dissemination of information to patients and their families. It was felt that digital platforms that offer a comprehensive guide to the diagnosis and its comorbidities, as well as reviewing treatment options and outcomes were of high value to parents and may improve children's compliance with treatment plans.

Engagement and education of different stakeholders, such as school staff, regarding AIS management and postoperative recovery was important. It was noted that some stakeholders may feel uncomfortable with children's capabilities and may restrict activities based on this assumption.

An essential component of this conference was the presentation by a patient and families that highlighted the impact the diagnosis of AIS had on them. The patient reported on how the diagnosis was suspected because of family members noting shoulder asymmetry and not being aware of the deformity themselves. The subsequent consultations with medical professionals, communication and disclosure of the diagnosis, the level of care received and the impact AIS had on their lives and their family were also described.

The parents of patients with AIS also reported their experiences with the communication of the diagnosis, their understanding of the information given, how this impacted their experience with the health care system, the frustration with the lack of a defined etiology that can form the basis of new therapies and the psychological impact the diagnosis had on them.

The early identification and stratification of the severity of scoliosis were quite important, as it allows early access to care to try and limit the progression or the deployment of interventions to correct the curve. Community-based screening of children was discussed and considered an essential process for early identification of scoliosis, although cost-effectiveness analyses noted in the literature showed cost at times to be prohibitive. Some methods to lower the costs of screening that were discussed included the development of innovative digital platforms and the use of new imaging technologies to capture spinal curving and assess its progression.

\section{Defining research priorities in AIS}

In discussions among group members, the priority areas that emerged to guide the research agenda included the identification of areas of scientific discovery to be prioritized and creating and assessing patient- and clinician-important 
outcome measures to evaluate the research impact on care and outcomes.

The discussions alluded to the challenges in defining effective interventions in the face of uncertainty of causative molecular mechanisms that can be targeted with specific therapies for AIS.

The priority areas identified by the group included studies related to natural history, mechanistic studies of spinal and paraspinal structures including non-invasive methodologies to assess tissues, the identification of clinical and biological markers of susceptibility and response to treatment, and the creation of robust preclinical and digital models that mimic the human disease to assess potential new therapies.

The study of scoliosis-related comorbidities was considered important. Pain was recognized as one of the high priority areas for exploration, especially trying to understand the mechanisms driving pain and therapeutic and prevention strategies that may help patients deal with this comorbidity. In addition, psychological effects of spinal curving and research on the quality of life of participants and their families was considered important.

\section{Define the structure of a national coalition to tackle AIS}

Roundtable discussions were conducted to define the structure of the national coalition. Participants were grouped with a mix of different stakeholders in each group. The questions revolved around potential participants, structure and sustainability of a coalition.

The group felt that having a multicenter coalition with a coordinating center is essential to allow the creation of an impactful structure. It will also allow research studies to proceed with the recruitment of a sufficiently large sample size for longitudinal observational studies and clinical trials.

The composition of the coalition should include multiple stakeholders from a diverse spectrum of interested individuals including patients, families, clinicians, basic science as well as translational and clinical researchers. Additional important participants should also include cost-effectiveness evaluators and policymakers, as they are involved in resource allocation that is imperative for the development and evaluation of research and clinical services.

Funding options were also considered, as this coalition will require significant resources to allow long-term sustainability for its functioning. The funding question was considered critical for success, and some center representatives were more hesitant to commit to this coalition initially because of this and wanted to see further expansion of the coalition and resource deployment prior to participation. However, at the end of the consultation process, the group affirmed its commitment to the creation of the national coalition to tackle AIS.

\section{Discussion}

AIS affects millions of adolescents globally. ${ }^{10,11}$ For such a common disease, relatively little progress has been made to understand its etiopathogenic mechanisms. The need for a large-scale effort to bring together the tools necessary to explore AIS mechanisms and improve outcomes was the driver for the creation of this national coalition. This coalition will be instrumental in creating new opportunities to target different aspects of care and outcomes in these patients.

Stakeholder discussions informed the priorities of AIS patients, including the involvement and engagement of patients and families in consultations about management and research priorities.

Over the past few years, patient- and family-important outcomes emerged as a central theme in patients' engagement with their health. ${ }^{12}$ Patients reported that defining their priorities in care was critical as a partnership, and that information sharing was a important piece of that engagement. This is an area that has been investigated in other fields of research, and there are few standardized approaches, including the Outcome Measures in Rheumatology that can be used as a model by the coalition to engage patients and families in the study and for care delivery. ${ }^{13}$

The role of the school was discussed concerning screening and as a place for educating teachers and carers about the needs of youth with AIS. Large-scale screening for scoliosis in school has demonstrated inconsistent results. ${ }^{14,15}$ In addition, the cost-effectiveness analyses have shown that this may not be feasible in all countries. ${ }^{16,17}$ However, this does not indicate that screening is inappropriate or not useful, as new methods that may make this feasible are worth exploring based on the discussions.

The structure of the coalition is a strength. Several stakeholders with diverse and complementary expertise are brought together, and this transdisciplinary approach has proven benefits regarding outcomes. ${ }^{18}$ The evaluation of the process of engagement of patients and families with researchers and clinicians in a highly specialized setting will be an important model to develop and allow strong partnerships to emerge. The funding for this coalition is indeed a major priority and involves the systematic development of funding efforts that will likely include grant submissions.

One of the priorities of this coalition is to guide researchers in a direction that families and patients deem 
necessary. The need for the identification of mechanistic and clinical research questions guided the prioritization of molecular biomarkers of susceptibility, initiation and progression of the curve, as well as the clinical questions including pain, physical health, psychological outcomes, and quality of life measures. These areas will be further developed.

Future directions will include the development of strategies for novel approaches to care, including the use of big data and machine learning to drive the identification of those at risk of scoliosis and developing approaches to help improve management.

While the specific purpose of this report is to describe the process and discussions that led to the development of the coalition, there are several important additional benefits to consider.

The process and structure of convening a meeting to discuss scoliosis is generalizable to other types of scoliosis, which are far less well characterized than AIS.

The conference model can also be applied to tackle other adolescent chronic diseases, and the development of a coalition is an important and feasible mechanism to study complex chronic diseases.

In addition, the inclusion of families and youth is a critical step to identifying patient-important outcomes and research priorities.

\section{Conclusion}

This meeting report provides valuable insight into what AIS patients and families consider important to them, as well as important targets that will help experts to shape study questions and interventions. The coalition stakeholders can implement these directions to improve the health outcomes of AIS patients.

\section{Acknowledgments}

We would like to thank the families who participated in this event. The conference was funded by the Canadian Institutes for Health Research (Grant number 354849).

\section{Author contributions}

All authors contributed to data analysis, drafting and revising the article, gave final approval of the version to be published, and agree to be accountable for all aspects of the work.

\section{Disclosure}

The authors report no conflicts of interest in this work.

\section{References}

1. Korbel K, Kozinoga M, Stoliński Ł, Kotwicki T. Scoliosis Research Society (SRS) criteria and Society of Scoliosis Orthopaedic and Rehabilitation Treatment (SOSORT) 2008 guidelines in non-operative treatment of idiopathic scoliosis. Pol Orthop Traumatol. 2014;79: $118-122$.

2. Misterska E, Glowacki M, Latuszewska J, Adamczyk K. Perception of stress level, trunk appearance, body function and mental health in females with adolescent idiopathic scoliosis treated conservatively: a longitudinal analysis. Qual Life Res. 2013;22(7):1633-1645.

3. Théroux J, May SL, Fortin C, Labelle H. Prevalence and management of back pain in adolescent idiopathic scoliosis patients: a retrospective study. Pain Res Manag. 2015;20(3):153-157.

4. Danielsson AJ, Wiklund I, Pehrsson K, Nachemson AL. Health-related quality of life in patients with adolescent idiopathic scoliosis: a matched follow-up at least 20 years after treatment with brace or surgery. Eur Spine J. 2001;10(4):278-288.

5. Pellegrino LN, Avanzi O. Prospective evaluation of quality of life in adolescent idiopathic scoliosis before and after surgery. J Spinal Disord Tech. 2014;27(8):409-414.

6. Weinstein SL, Dolan LA, Wright JG, Dobbs MB. Effects of bracing in adolescents with idiopathic scoliosis. N Engl J Med. 2013;369(16): 1512-1521.

7. Bettany-Saltikov J, Weiss HR, Chockalingam N, et al. Surgical versus non-surgical interventions in people with adolescent idiopathic scoliosis. Cochrane Database Syst Rev. 2015;4:CD010663.

8. Wajchenberg M, Astur N, Kanas M, Martins DE. Adolescent idiopathic scoliosis: current concepts on neurological and muscular etiologies. Scoliosis Spinal Disord. 2016;11:4.

9. Latalski M, Danielewicz-Bromberek A, Fatyga M, Latalska M, Kröber M, Zwolak P. Current insights into the aetiology of adolescent idiopathic scoliosis. Arch Orthop Trauma Surg. 2017;137(10):1327-1333.

10. Weinstein SL, Dolan LA, Cheng JC, Danielsson A, Morcuende JA. Adolescent idiopathic scoliosis. Lancet. 2008;371(9623): $1527-1537$.

11. Konieczny MR, Senyurt H, Krauspe R. Epidemiology of adolescent idiopathic scoliosis. J Child Orthop. 2013;7(1):3-9.

12. Tubach F, Ravaud $P$, Baron G, et al. Evaluation of clinically relevant changes in patient reported outcomes in knee and hip osteoarthritis: the minimal clinically important improvement. Ann Rheum Dis. 2005;64(1):29-33.

13. Goldsmith CH, Boers M, Bombardier C, Tugwell P. Criteria for clinically important changes in outcomes: development, scoring and evaluation of rheumatoid arthritis patient and trial profiles. OMERACT Committee. J Rheumatol. 1993;20(3):561-565.

14. Luk KD, Lee CF, Cheung KM, et al. Clinical effectiveness of school screening for adolescent idiopathic scoliosis: a large population-based retrospective cohort study. Spine. 2010;35(17):1607-1614.

15. Fong DY, Lee CF, Cheung KM, et al. A meta-analysis of the clinical effectiveness of school scoliosis screening. Spine. 2010;35(10):1061-1071.

16. Lee CF, Fong DY, Cheung KM, et al. Costs of school scoliosis screening: a large, population-based study. Spine. 2010;35(26): 2266-2272.

17. Yawn BP, Yawn RA. The estimated cost of school scoliosis screening. Spine. 2000;25(18):2387-2391.

18. Disis ML, Slattery JT. The road we must take: multidisciplinary team science. Sci Transl Med. 2010;222(22):22cm9. 


\section{Publish your work in this journal}

Adolescent Health, Medicine and Therapeutics is an international, peer-reviewed, open access journal focusing on health, pathology, and treatment issues specific to the adolescent age group. All aspects of health maintenance, preventative measures and disease treatment interventions are addressed within the journal and practitioners from all disciplines are invited to submit their work as well as healthcare researchers and patient support groups. This journal is included in PubMed. The manuscript management system is completely online and includes a very quick and fair peer-review system. Visit http://www.dovepress.com/testimonials. php to read real quotes from published authors.

Submit your manuscript here: http://www.dovepress.com/adolescent-health-medicine-and-therapeutics-journal 